\title{
A proteomic analysis identifies candidate early biomarkers to predict ovarian hyperstimulation syndrome in polycystic ovarian syndrome patients
}

\author{
LAN WU ${ }^{1,2^{*}}$, YAZHOU SUN ${ }^{3 *}, J_{U N} W_{A N}^{2}$, TING LUAN $^{2}$, QING CHENG $^{2}$ and YONG TAN ${ }^{1}$ \\ ${ }^{1}$ First Clinical Medicine College, Nanjing University of Chinese Medicine, Nanjing, Jiangsu 210046; ${ }^{2}$ Department of \\ Obstetrics, Nanjing Medical University Affiliated Nanjing Maternal and Child Health Hospital, Nanjing, Jiangsu 210004; \\ ${ }^{3}$ Department of Pediatrics, The Third Affiliated Hospital of Xinxiang Medical University, Xinxiang, Henan 453003, P.R. China
}

Received April 5, 2016; Accepted March 8, 2017

DOI: $10.3892 / \mathrm{mmr} .2017 .6604$

\begin{abstract}
Ovarian hyperstimulation syndrome (OHSS) is a potentially life-threatening, iatrogenic complication that occurs during assisted reproduction. Polycystic ovarian syndrome (PCOS) significantly increases the risk of OHSS during controlled ovarian stimulation. Therefore, a more effective early prediction technique is required in PCOS patients. Quantitative proteomic analysis of serum proteins indicates the potential diagnostic value for disease. In the present study, the authors revealed the differentially expressed proteins in OHSS patients with PCOS as new diagnostic biomarkers. The promising proteins obtained from liquid chromatography-mass spectrometry were subjected to ELISA and western blotting assay for further confirmation. A total of 57 proteins were identified with significant difference, of which 29 proteins
\end{abstract}

Correspondence to: Professor Qing Cheng, Department of Obstetrics, Nanjing Medical University Affiliated Nanjing Maternal and Child Health Hospital, 123 Tianfei Lane, Mochou Road, Nanjing, Jiangsu 210004, P.R. China

E-mail: chengqing2016@163.com

Professor Yong Tan, First Clinical Medicine College, Nanjing University of Chinese Medicine, 282 Hanzhong Road, Nanjing, Jiangsu 210046, P.R. China

E-mail: gdtotp1@sina.com

${ }^{*}$ Contributed equally

Abbreviations: IVF, in vitro fertilization; OHSS, ovarian hyperstimulation syndrome; COS, controlled ovarian stimulation; PCOS, polycystic ovarian syndrome; E2, estradiol; RAS, renin-angiotensin system; $\mathrm{LH}$, luteinizing hormone; $\mathrm{FSH}$, follicle stimulating hormone; BMI, body mass index; AFC, antral follicle count; $\mathrm{AMH}$, anti-müllerian hormone; ROC, receiver operating characteristic; GO, gene ontology; COG, clusters of orthologous groups of proteins; $\mathrm{T}$, testosterone

Key words: polycystic ovary syndrome, ovarian hyperstimulation syndrome, proteomics, serum biomarkers were upregulated and 28 proteins were downregulated in OHSS patients. Haptoglobin, fibrinogen and lipoprotein lipase were selected as candidate biomarkers. Receiver operating characteristic curve analysis demonstrated all three proteins may have potential as biomarkers to discriminate OHSS in PCOS patients. Haptoglobin, fibrinogen and lipoprotein lipase have never been reported as a predictive marker of OHSS in PCOS patients, and their potential roles in OHSS occurrence deserve further studies. The proteomic results reported in the present study may gain deeper insights into the pathophysiology of OHSS.

\section{Introduction}

Although in vitro fertilization (IVF) is a safe and common treatment approach for infertility, there still remain some undesired side effects. As a serious iatrogenic complication, ovarian hyperstimulation syndrome (OHSS) is encountered during controlled ovarian stimulation (COS). Classification of OHSS is based on laboratory findings and clinical characteristics. The mild forms of OHSS are common, occurring in up to $30 \%$ of all IVF cycles, while the incidence of moderate and severe forms was 3-6\% and $0.2-1 \%$ of IVF cycles, respectively $(1,2)$. As the most severe complication of COS, OHSS is characterized by a dramatic ovarian enlargement and an acute shift of intravascular fluid, which is caused by increased vascular permeability and ovarian neoangiogenesis (3). Severe forms can cause thrombus formation (4), and can even become life threatening. However, despite many years of clinical experience, the pathogenesis of OHSS remains ambiguous.

Risk factors that relate to the development of OHSS include young age, low body weight, PCOS, previous episodes of OHSS, multiple pregnancy, rapidly rising estradiol (E2) levels and follicle number (>20-25) (5-7). Serum levels of vascular endothelial growth factor (VEGF) in follicular fluid, pleural effusion and ascites are higher in OHSS patients compared with healthy people (8). VEGF may serve as an inflammatory factor to increase vascular permeability and neovascularization, which may explain the clinical symptoms of OHSS. Ingman and Robertson (9) suggested that human chorionic gonadotropin (hCG) participates in OHSS by activating the 
renin-angiotensin system (RAS). The ovarian RAS is involved in regulating endothelial proliferation, vascular permeability, angiogenesis and prostaglandin release (10). The increase of renin, angiotensin II and angiotensin-converting enzyme also lead to pathological changes in OHSS patients. The roles of genetic predisposition, luteinizing hormone ( $\mathrm{LH}$ ), inflammatory mediators and follicle stimulating hormone (FSH) variability are already discussed in the pathophysiology of OHSS (1-3,11). However, there is still a lack of an effective approach for the prevention and treatment of OHSS as the pathogenesis has not yet been fully elucidated.

Prevention is often better than cure. However, using only body mass index (BMI) and age to predict OHSS prior to $\mathrm{COH}$ remains an arduous task in an individual IVF cycle. Monitoring the serum E2 level and antral follicle count (AFC) are conventional means for OHSS prevention (11). Serum hormonal markers, such as anti-müllerian hormone (AMH) are increasingly being applied to predict ovarian response to stimulation (12). However, these diagnosis indices cannot accurately predict OHSS prior to starting $\mathrm{COH}$, a better alternative for early diagnosis is needed. Now, proteomics have been widely used to discover biomarkers for various diseases. Markel et al (13) reported that CEACAM1 and MICA could serve as novel serum biomarkers in patients with acute and recurrent pericarditis. Xu et al (14) demonstrated that the candidate serum biomarkers (S100A9, SOD3 and MMP9) performed high sensitivity and specificity in discriminating pulmonary tuberculosis. These findings indicate that serum protein biomarkers serve an important role in the prediction and diagnosis of diseases. Therefore, the authors hypothesize that serum proteins may serve as effective biomarkers for OHSS prediction. Thus, there is a need for a comprehensive analysis on OHSS patient's serum proteins for predicting and preventing OHSS.

As a proven well-known factor of OHSS in all populations, PCOS is a common endocrine disorder syndrome that leads to infertility in child-bearing age women, with a prevalence of $5-10 \%$ (15). In the present study, the authors compared the expression profiles of serum protein in OHSS patients and non-OHSS patients with PCOS through iTRAQ-coupled liquid chromatography-mass spectrometry (LC-MS) before $\mathrm{COH}$ was initiated, in order to reveal the potential markers. Three candidate protein biomarkers (haptoglobin, lipoprotein lipase and fibrinogen) were validated by ELISA and western blotting. Further receiver operating characteristic (ROC) curves analysis of these biomarkers presented sufficient predictive values for clinical applications. The potential implications of clinical symptoms for OHSS precaution were also discussed.

\section{Materials and methods}

Study design and population. The authors conducted the present study in the Nanjing Maternity and Child Health Care Hospital (Nanjing, China). The research was approved by the Human Research Ethics Committee of Nanjing Medical University (Nanjing, China) and informed consent was provided by each participant prior to research. A total of 88 patients who suffered from PCOS and were undergoing IVF treatment were recruited. The diagnosis of PCOS was in accordance with the Rotterdam criteria revised in 2003; the patient should suffer with at least two of the three indicators: (A) Menstruation or non-ovulation; (B) clinical and/or biochemical display hyperandrogenism; (C) ultrasound examination presented polycystic ovaries, and exclusion of other diseases, such as adrenal hyperplasia, Cushing's syndrome, androgen-secreting tumors and hyperprolactinemia. Diane-35 had been used in all the patients who have PCOS to let the serum testosterone levels decrease to normal. Blood samples were obtained prior to gonadotropin injection and were centrifuged at $1,000 \mathrm{x} \mathrm{g}$ at $4^{\circ} \mathrm{C}$ for 10 min to obtain the sera and then stored at $-80^{\circ} \mathrm{C}$ for further use.

Patients involved in the present study were divided into two groups according to the results of $\mathrm{COH}$ : Women who develop moderate or severe OHSS were selected as the 'OHSS group' and others without OHSS or with mild OHSS were assigned to the 'control group'. Firstly, the mixed serum of ten women from each group to identified the altered proteins through analysis by LC-MS/MS. The authors revealed the expression levels of serum proteins and selected significantly different expression proteins in the OHSS group for further analysis. Next, the screened proteins were validated in the other samples by ELISA and western blotting.

Classification of OHSS. The classification of OHSS was defined by Golan and Weissman (16). The following features were classified as moderate OHSS: Stomach pains and discomfort, abdominal distension, nausea, ascites and the size of ovary $(8-12 \mathrm{~cm})$. Severe OHSS were characterized by massive ascites, hydrothorax, $45 \%$ hematocrit edema/anasarca, liver dysfunction, and white blood cell count $>15,000$.

Protein preparation and LC-MS/MS analysis. The depletion of abundant serum proteins was carried out using ProteoMiner Protein Enrichment kit (Bio-Rad Laboratories, Inc., Hercules, CA, USA), so as to identify more low abundance proteins, according to the manufacturer's protocol. Then, $100 \mu \mathrm{g}$ total protein from each mixed sample was taken out and incubated with Trypsin Gold (Promega Corporation, Madison, WI, USA). Next, the peptides were labeled with the iTRAQ reagents (Applied Biosystems; Thermo Fisher Scientific, Inc., Waltham, MA, USA). Strong cation exchange (SCX) chromatography was conducted by a high-performance liquid chromatography pump system LC-20AB (Shimadzu, Kyoto, Japan). The iTRAQ-labeled peptide mixtures were dissolved and then loaded onto a Ultremex SCX column (Phenomenex, Torrance, CA, USA; $4.6 \times 250 \mathrm{~mm}$ ). The fractions collected from SCX chromatography were performed MS/MS analysis.

Validation by ELISA and western blotting. Fold changes of candidate biomarkers were further validated by ELISA and western blotting techniques. A total of 68 serum samples were screened, including 38 OHSS women and 30 controls. ELISA quantifications for haptoglobin, fibrinogen and lipoprotein lipase (Abcam, Cambridge, MA, USA) were performed according to the manufacturers' instructions of commercial ELISA kits. For western blotting, $30 \mu \mathrm{g}$ serum proteins were electrophoretically separated on a $10-12 \%$ SDS-PAGE gel and then transferred to polyvinylidene difluoride membranes (EMD Millipore, Billerica, MA, USA). 
Table I. Comparison of clinical characteristics between patients with OHSS and without OHSS.

\begin{tabular}{|c|c|c|c|}
\hline & OHSS $(n=40)$ & Control $(n=48)$ & P-value \\
\hline Age (years) & $28.1 \pm 3.8$ & $28.5 \pm 3.2$ & NS \\
\hline Body mass index $\left(\mathrm{kg} / \mathrm{m}^{2}\right)$ & $21.3 \pm 2.6$ & $24.3 \pm 3.7$ & $<0.001$ \\
\hline Fasting insulin (mU/l) & $14.7 \pm 2.5$ & $13.8 \pm 3.6$ & NS \\
\hline Infertility duration (years) & $3.5 \pm 2.5$ & $3.7 \pm 2.1$ & NS \\
\hline Basal serum FSH (U/l) & $6.1 \pm 1.8$ & $6.4 \pm 1.7$ & NS \\
\hline Basal serum LH (U/l) & $7.7 \pm 3.9$ & $5.3 \pm 2.4$ & $<0.01$ \\
\hline Basal LH/FSH (L) & $1.2 \pm 0.5$ & $0.81 \pm 0.32$ & $<0.01$ \\
\hline Basal serum E2 (U/1) & $43.5 \pm 18.1$ & $42.3 \pm 25.7$ & NS \\
\hline Basal serum T (ng/ml) & $0.53 \pm 0.14$ & $0.49 \pm 0.17$ & NS \\
\hline Dose of gonadotrophins (U) & $1423.5 \pm 342.3$ & $1617.3 \pm 456.1$ & NS \\
\hline Oestradiol on day of $\mathrm{HCG}(\mathrm{pg} / \mathrm{ml})$ & $10278.3 \pm 2654.2$ & $3324.4 \pm 953.4$ & $<0.001$ \\
\hline No. of oocytes & $17.9 \pm 2.4$ & $7.8 \pm 2.5$ & $<0.001$ \\
\hline No. of good embryos & $10.7 \pm 7.9$ & $4.5 \pm 2.6$ & $<0.001$ \\
\hline
\end{tabular}

OHSS, ovarian hyperstimulation syndrome; NS, not significant; LH, luteinizing hormone; FSH, follicle stimulating hormone; E2, estradiol; T, testosterone.

Following blocking with 5\% skimmed milk, membranes were incubated with the primary antibodies at the indicated dilutions: anti-haptoglobin $(1: 10,000$; cat no. ab13429; Abcam), anti-fibrinogen (1:10,000; cat no. ab119948; Abcam) and anti-lipoprotein lipase (1:1,000; cat no. ab21356; Abcam) at $4^{\circ} \mathrm{C}$ overnight. The blots were then washed with TBS containing $0.05 \%$ Tween-20 and incubated with horseradish peroxidase (HRP)-conjugated secondary antibody $(1: 10,000$; cat no. 31439; Thermo Fisher Scientific, Inc.) at room temperature for $2 \mathrm{~h}$. Immunopositive bands were visualized using Luminata Western HRP Substrates (EMD Millipore) and densitometry of the bands were estimated by FluorChem M system (ProteinSimple, San Jose, CA, USA).

Data and bioinformatics analysis. Protein identification and quantification were performed by using the Mascot search engine (Matrix Science, Ltd., London, UK). These proteins with a fold changes of $>1.2$ or $<0.83$ (OHSS vs. control) were considered as significant $(\mathrm{P}<0.05)$. Functional annotations of the proteins were conducted using Gene Ontology (GO) database (http://geneontology.org/), which can describe cellular component, molecular function and biological process, respectively. The Cluster of Orthologous Groups (COGs) database (http://www.ncbi.nlm.nih.gov/COG/) was used to group and classify all the proteins identified in both groups. Every protein in COG is supposed to derive from the same protein ancestor.

Statistical analysis. All statistical analyses of this study were performed using SPSS software (version 20.0.0; IBM SPSS, Armonk, NY, USA). Data were presented as mean \pm standard deviation. Inter-group differences in the clinical characteristics and experimental data were estimated by the Student's $\mathrm{t}$-test and $\mathrm{P}<0.05$ was considered to indicate a statistically significant difference. ROC curves were generated to evaluate the diagnostic value of candidate biomarkers.

\section{Results}

Characteristics of the study population. A total of 88 PCOS patients were enrolled in our study and 40 had confirmed OHSS, their characteristics were summarized in Table I. As indicated, there was no difference between the two groups on age, basal E2, basal testosterone, basal FSH, fasting insulin, duration of infertility and gonadotropin dose $(\mathrm{P}>0.05)$. The values of basal LH, basal LH/FSH, number of oocytes and number of good embryos were significantly higher in the OHSS group than in the control group $(\mathrm{P}<0.05)$, whereas the values of BMI and estradiol on the hCG day were significantly lower in the OHSS group than in the control group $(\mathrm{P}<0.05)$.

Identification of differentially expressed serum proteins in women with OHSS. To identify potential biomarkers of OHSS, 20 serum samples from OHSS and control groups were divided into four main groups with five samples from each group. The mixtures were employed using iTRAQ combined with LC-MS/MS technologies. A total of 418 unique proteins were identified by MS in both groups. A total of 57 significant different proteins were screened in OHSS serum samples, compared with the control group, of which 28 proteins were overexpressed ( $>1.2$-fold) and 29 proteins were underexpressed (<0.83-fold; Fig. 1). The full list of differentially expressed proteins can be identified in Table II.

Functional classification of identified proteins. The authors categorized these identified proteins according to their biological functions using $\mathrm{GO}$ analysis. The promising biological functions are presented in Fig. 2A-C. Cellular component analysis demonstrated that these proteins were mainly enriched in membrane (13.7\%), organelle (19.1\%) and extracellular regions $(19.6 \%)$. According to the analysis of molecular function, proteins were categorized into groups of 

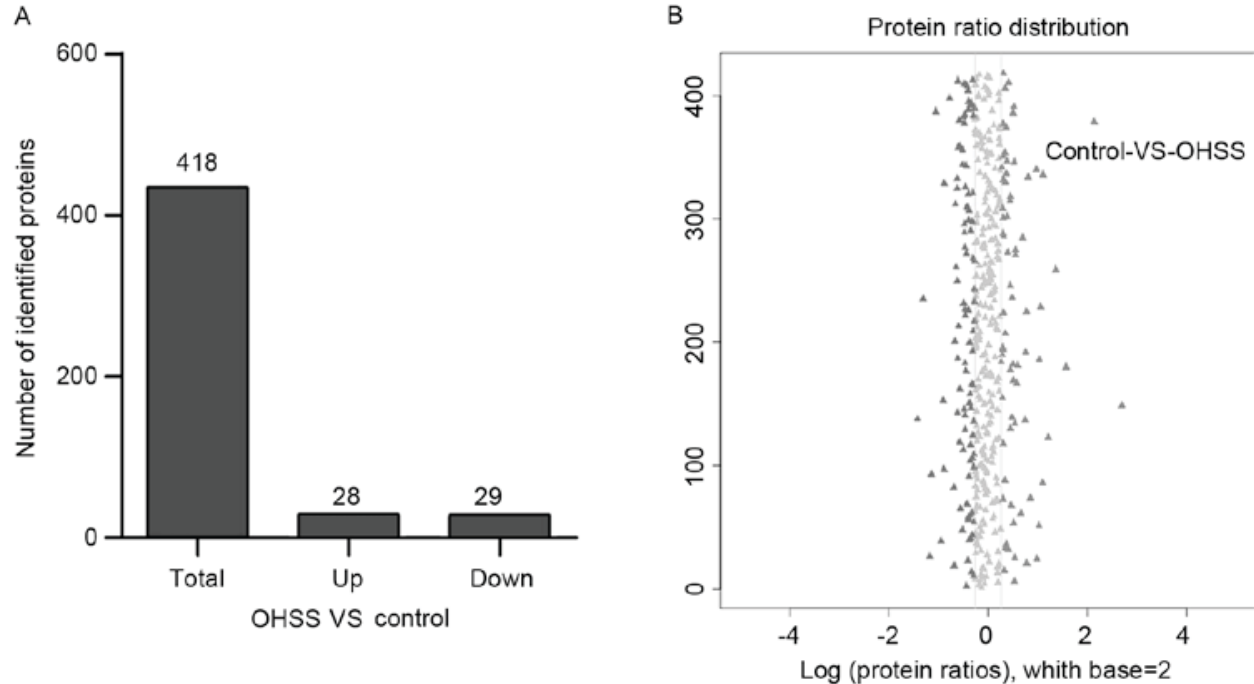

Figure 1. The identified proteins in polycystic ovary syndrome women who develop moderate or severe OHSS and control group. (A) Statistics of differentially expressed proteins in both groups. (B) Abundance analysis presented a distinguishable protein profiling among groups. When the fold changes $>1.2$ and $\mathrm{P}<0.05$, the protein was considered as significantly different protein. OHSS, ovarian hyperstimulation syndrome.

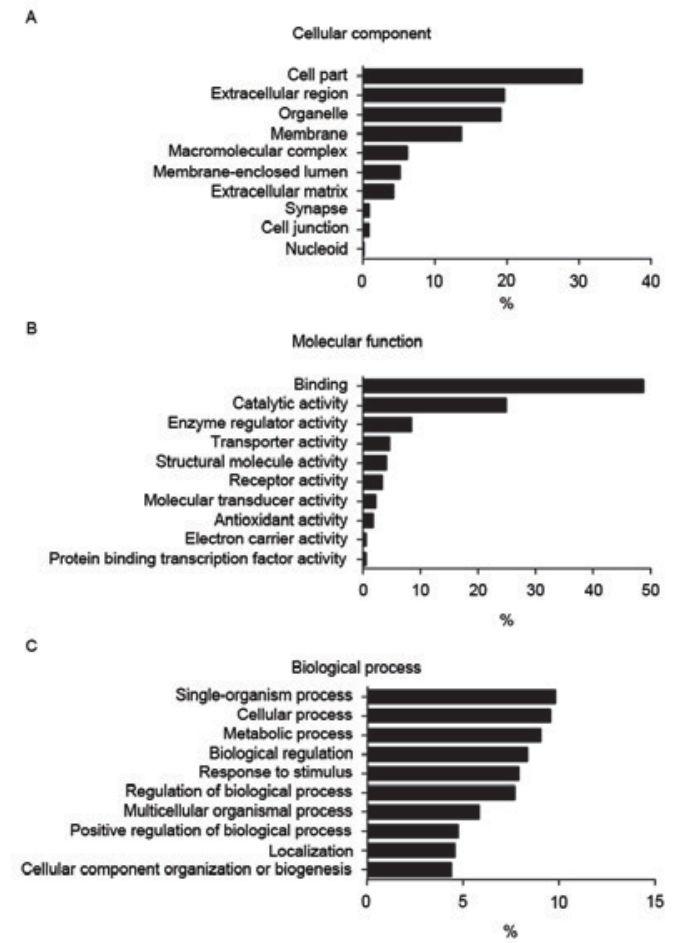

D

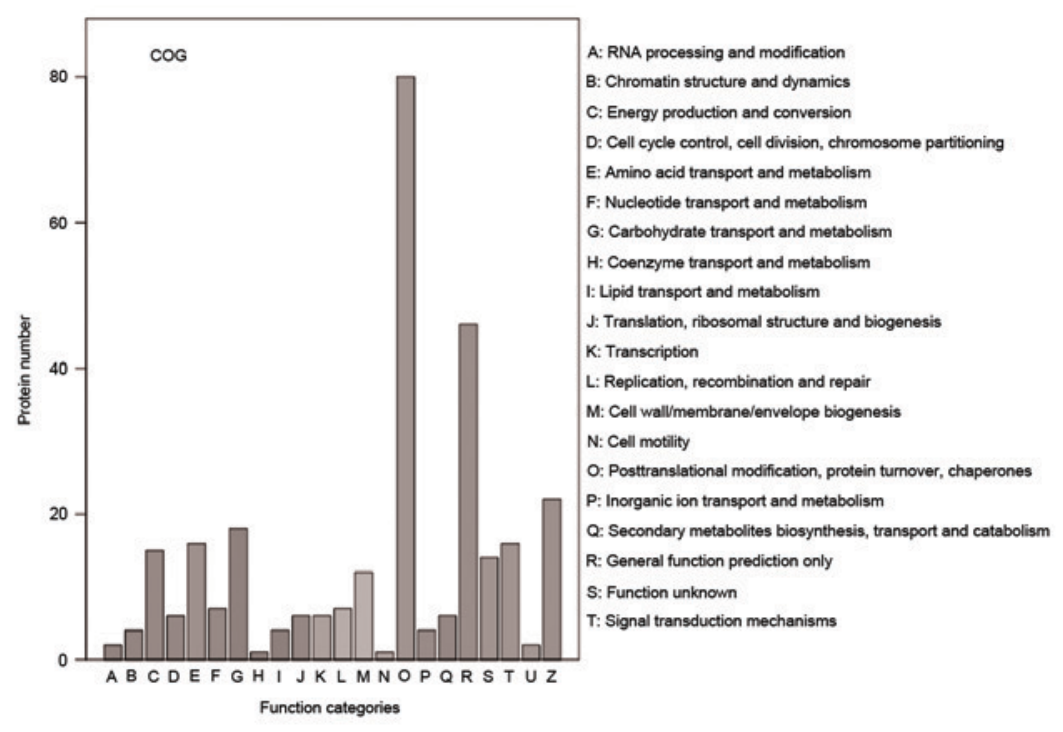

Figure 2. Gene Ontology and COG analysis of identified proteins. The following promising biological functions are presented in the figure: (A) Cellular component, (B) molecular function, (C) biological process and (D) COG classification. COG, Clusters of Orthologous Groups of proteins.

enzyme regulator activity $(8.4 \%)$, catalytic activity $(25.0 \%)$ and binding $(48.7 \%)$. The identified proteins involved in diverse biological processes, such as regulation of biological process $(7.7 \%)$, response to stimulus $(7.9 \%)$, biological regulation $(8.3 \%)$, metabolic process $(9.0 \%)$, cellular process $(9.5 \%)$ and single-organism process $(9.8 \%)$. COG analysis indicated the participation of identified proteins in a diverse number of biological processes, including posttranslational modification, cytoskeleton, protein turnover chaperones, lipid transport and metabolism (Fig. 2D).
Validation of candidate biomarkers. Based on bioinformatics analysis, the authors selected three abnormally expressed proteins between two groups for further study, haptoglobin, fibrinogen and lipoprotein lipase. These three proteins involved in inflammatory responses, immune responses and angiogenesis, which are the pathogenic basis of OHSS. The ratios of haptoglobin, lipoprotein lipase and the fibrinogen subunit (alpha chain, beta chain and gamma chain) were 0.48 , $2.31,1.48,1.71$ and 1.53 , respectively, compared to the control group (Table II). Verification of those candidate biomarkers 
Table II. List of proteins with significant difference in levels between OHSS and control groups.

\begin{tabular}{|c|c|c|c|}
\hline Accession no. & Protein name & MW (kDa) & Ratio (OHSS/control) \\
\hline P30043 & Flavin reductase & 25,262 & 2.46 \\
\hline P49060 & Lipoprotein lipase & 63,225 & 2.31 \\
\hline P78371 & T-complex protein 1 subunit beta & 69,354 & 1.92 \\
\hline P0DJI8 & Serum amyloid A-1 protein & 15,436 & 1.87 \\
\hline P08603 & Complement factor $\mathrm{H}$ & 168,175 & 1.84 \\
\hline P02675 & Fibrinogen beta chain & 48,076 & 1.71 \\
\hline Q16610 & Extracellular matrix protein 1 & 20,792 & 1.60 \\
\hline P06732 & Creatine kinase M-type & 54,181 & 1.53 \\
\hline P01788 & $\mathrm{Ig}$ heavy chain $\mathrm{V}$ region $\mathrm{H} 8$ & 13,100 & 1.53 \\
\hline P02679 & Fibrinogen gamma chain & 63,883 & 1.53 \\
\hline P67936 & Tropomyosin alpha-4 chain & 33,316 & 1.52 \\
\hline P68872 & Hemoglobin subunit beta & 14,272 & 1.52 \\
\hline Q9UBX5 & Fibulin-5 & 19,441 & 1.50 \\
\hline P02671 & Fibrinogen alpha chain & 109,041 & 1.48 \\
\hline Q13790 & Apolipoprotein $\mathrm{F}$ & 39,039 & 1.42 \\
\hline Q08380 & Galectin-3-binding protein & 72,286 & 1.40 \\
\hline P04114 & Apolipoprotein B-100 & 222,441 & 1.38 \\
\hline P04206 & Ig kappa chain V-III region GOL & 13,154 & 1.37 \\
\hline P01599 & Ig kappa chain V-I region Gal & 11,818 & 1.35 \\
\hline P0CG05 & Ig lambda- 2 chain $\mathrm{C}$ regions & 27,347 & 1.35 \\
\hline P01743 & Ig heavy chain V-I region HG3 & 19,786 & 1.34 \\
\hline Q9HDC9 & Adipocyte plasma membrane-associated protein & 50,834 & 1.34 \\
\hline P23381 & Tryptophan-tRNA ligase, cytoplasmic & 64,425 & 1.32 \\
\hline P01591 & Immunoglobulin J chain & 21,450 & 1.31 \\
\hline Q9CWF2 & Tubulin beta-2B chain & 46,571 & 1.31 \\
\hline O60462 & Neuropilin-2 & 115,991 & 1.31 \\
\hline P04431 & Ig kappa chain V-I region Walker & 14,314 & 1.30 \\
\hline Q9UHG3 & Prenylcysteine oxidase 1 & 63,740 & 1.22 \\
\hline P01042 & Kininogen-1 & 57,836 & 0.81 \\
\hline P04264 & Keratin, type II cyt & 75,006 & 0.81 \\
\hline P43652 & Afamin & 86,781 & 0.80 \\
\hline P01011 & Alpha-1-antichymotrypsin & 59,285 & 0.79 \\
\hline P06310 & Ig kappa chain V-II region RPMI-6410 & 14,040 & 0.79 \\
\hline P04217 & Alpha-1B-glycoprotein & 58,440 & 0.78 \\
\hline Q9NZP8 & Complement $\mathrm{C} 1 \mathrm{r}$ subcomponent-like protein & 59,681 & 0.78 \\
\hline P02774 & Vitamin D-binding protein & 67,663 & 0.77 \\
\hline P05543 & Thyroxine-binding globulin & 55,459 & 0.77 \\
\hline P00740 & Coagulation factor IX & 61,901 & 0.77 \\
\hline Q13275 & Semaphorin-3F & 86,066 & 0.76 \\
\hline Q96IY4 & Carboxypeptidase B2 & 51,257 & 0.75 \\
\hline P26927 & Hepatocyte growth factor-like protein & 91,508 & 0.73 \\
\hline P0C0L5 & Complement C4-B & 43,077 & 0.71 \\
\hline P10643 & Complement component C7 & 111,446 & 0.71 \\
\hline P07996 & Thrombospondin-1 & 150,327 & 0.70 \\
\hline Q6Q759 & Sperm-associated antigen 17 & 150,152 & 0.70 \\
\hline P25311 & Zinc-alpha-2-glycoprotein & 40,854 & 0.70 \\
\hline P08571 & Monocyte differentiation antigen CD14 & 43,702 & 0.70 \\
\hline P06396 & Gelsolin & 96,753 & 0.69 \\
\hline Q5R767 & SPARC & 43,328 & 0.68 \\
\hline P00751 & Complement factor B & 168,440 & 0.68 \\
\hline P36980 & Complement factor $\mathrm{H}$-related protein 2 & 34,920 & 0.68 \\
\hline P02763 & Alpha-1-acid glycoprotein 1 & 28,288 & 0.67 \\
\hline
\end{tabular}


Table II. Continued.

\begin{tabular}{llcc}
\hline Accession no. & \multicolumn{1}{c}{ Protein name } & MW (kDa) & Ratio (OHSS/control) \\
\hline P00352 & Retinal dehydrogenase 1 & 56,272 & 0.59 \\
P02787 & Serotransferrin & 97,259 & 0.58 \\
P02768 & Serum albumin & 89,874 & 0.55 \\
P00738 & Haptoglobin & 56,812 & 0.48 \\
P00325 & Alcohol dehydrogenase 1B & 49,155 & 0.43
\end{tabular}

MW, molecular weight; OHSS, ovarian hyperstimulation syndrome.

A

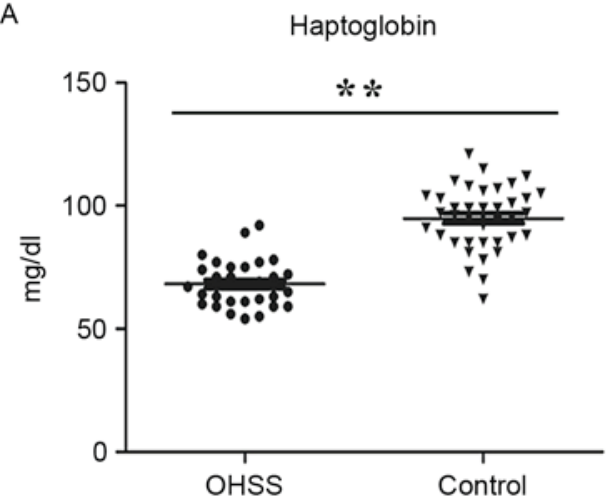

C

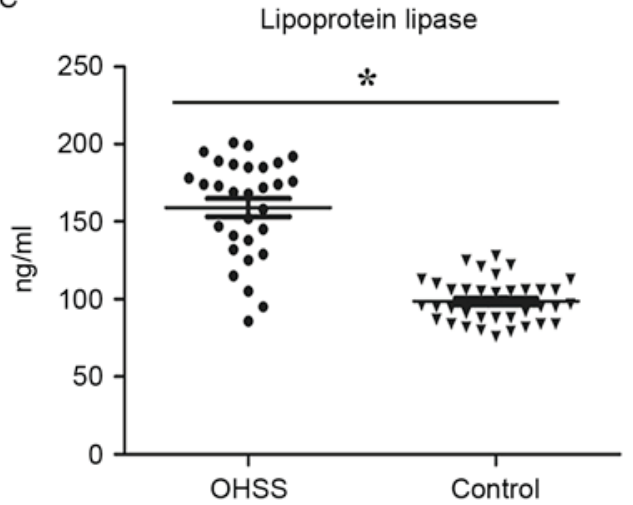

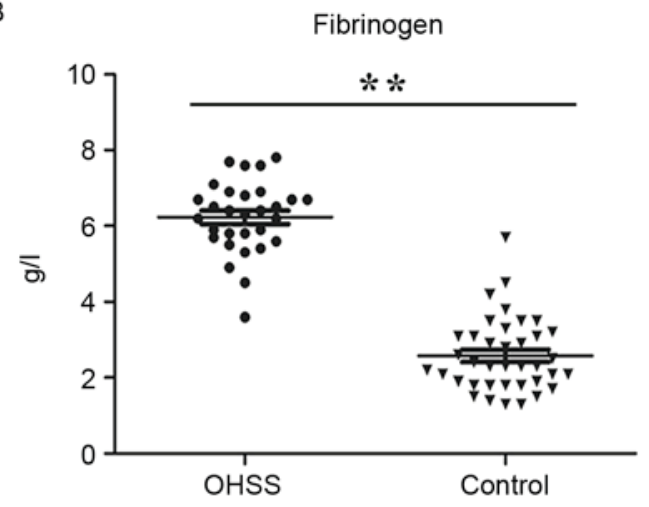

D

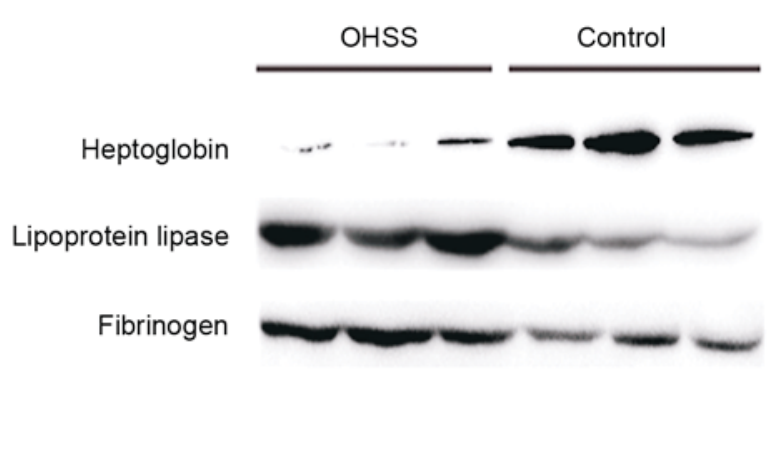

Figure 3. Validation the expression of candidate proteins in serum samples. ELISA results of (A) haptoglobin, (B) fibrinogen and (C) lipoprotein lipase in the OHSS group $(n=40)$ and normal controls $(n=48)$. P-values were calculated with Student's t-test. ${ }^{* *} \mathrm{P}<0.01$, ${ }^{*} \mathrm{P}<0.05$ OHSS vs. control. (D) The western blotting validated the relative expression of haptoglobin, fibrinogen and lipoprotein lipase in serum samples. OHSS, ovarian hyperstimulation syndrome.

was performed by ELISA assay and western blotting in a large and independent cohort. In accordance with the result from MS analysis, haptoglobin was significantly downregulated in OHSS patients, while lipoprotein lipase and fibrinogen were upregulated, compared with the control group (Fig. 3).

Prediction value of candidate biomarkers for OHSS. ROC curve analysis revealed that the relative expression level of haptoglobin, fibrinogen and lipoprotein lipase in serum performed more accurately for OHSS prediction. Specificity, sensitivity and the area under the curve (AUC) value of haptoglobin were $0.875,0.933,0.913$, respectively (Fig. 4A). When fibrinogen individually served as a biomarker, specificity, sensitivity and AUC value was 0.811, 0.937 and 0.925, respectively (Fig. 4B). In addition, lipoprotein lipase may discriminate OHSS from control patients; the specificity, sensitivity and AUC value were 0.775, 0.907 and 0.882 , respectively (Fig. 4C). The combination of all three proteins resulted in an AUC of 0.917 , and the sensitivity and specificity were 0.882 and 0.923 , respectively (Fig. 4D). Furthermore, the authors evaluated the discriminating effect of the basal $\mathrm{LH} / \mathrm{FSH}$ ratio and BMI for the prediction of OHSS, the AUC value of LH/FSH ratio and BMI were 0.741 and 0.729 (Fig. 4E and F). The sensitivity and specificity of the basal LH/FSH ratio were 0.709 and 0.821 , and $\mathrm{BMI}$ were 0.833 and 0.787 , respectively (Fig. 4E and F). 

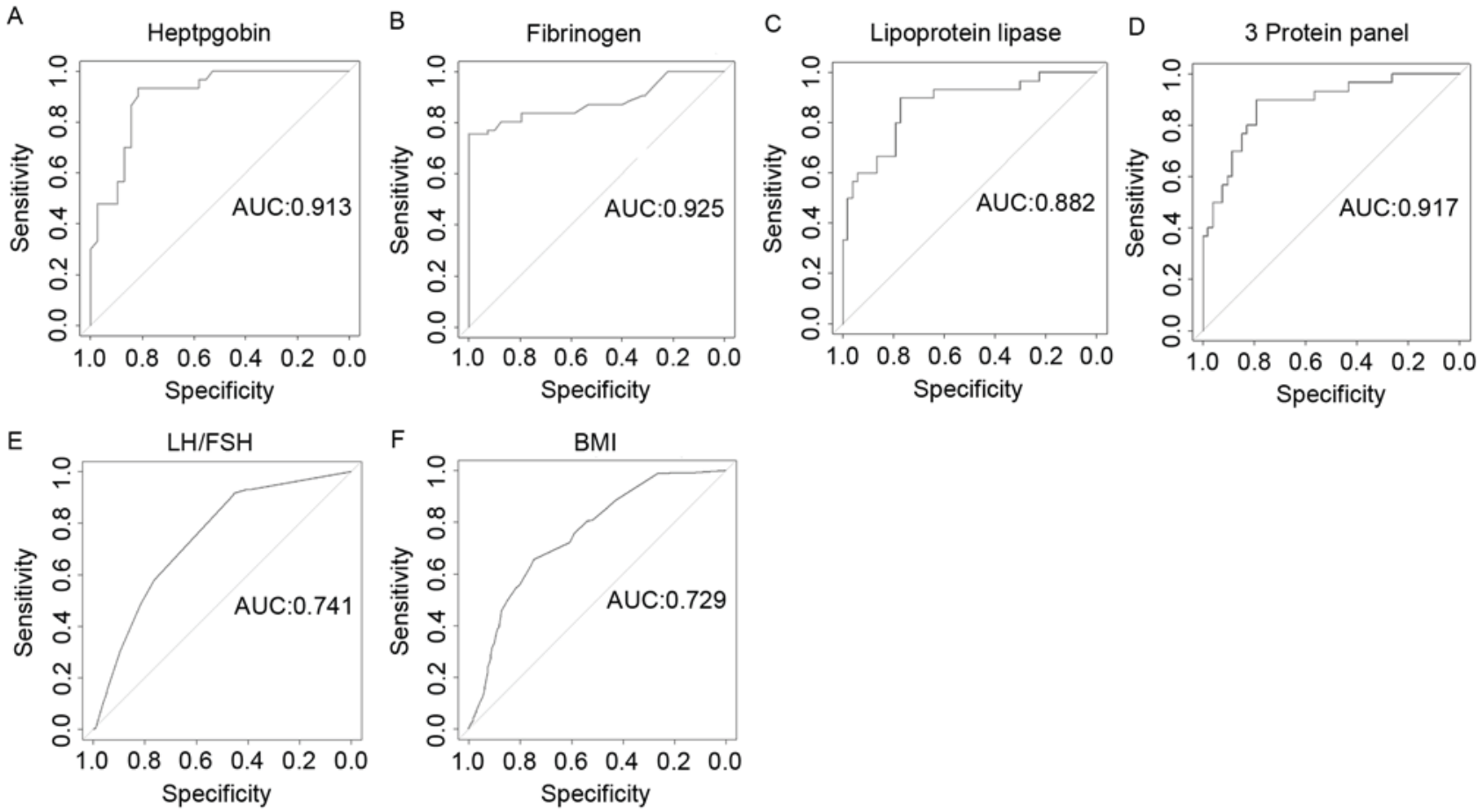

Figure 4. Efficacy of haptoglobin, fibrinogen, lipoprotein, LH/FSH and BMI in distinguishing ovarian hyperstimulation syndrome patients from normal polycystic ovary syndrome controls. ROC curve analysis of (A) haptoglobin, (B) fibrinogen (B and C) lipoprotein lipase and (D) a combinations using ELISA assays. (E and F) ROC curve using clinical characteristics. LH/FSH and BMI. AUC is indicated in each plot. LH, luteinizing hormone; FSH, follicle stimulating hormone; BMI, body mass index; ROC, receiver operating characteristic; AUC, area under the curve.

\section{Discussion}

As the most common endocrine disorder, PCOS is a clear risk factor for OHSS in reproductive-age women. However, the specific etiology of OHSS in PCOS patients is still poorly understood, its accurate prediction in an individual IVF cycle is difficult. OHSS often occurs in the absence of currently reported risk factors, so a more effective early prevention measure is needed. Comparative proteomic analysis has been widely used in screening promising diagnosis biomarkers for diseases (17-19). The present study attempted to screen candidate diagnostic serum proteins in OHSS patients with PCOS. A total of 57 significantly different proteins were identified and three significant different proteins, including haptoglobin, fibrinogen and lipoprotein lipase were validated by ELISA and western blotting. Finally, the predictive value of these three candidate biomarkers was further evaluated. To the best of the authors' knowledge, this is the first expression pattern study of serum proteins in PCOS women with or without severe OHSS.

Age, BMI, hormonal markers, as well as infertility cause in general were considered as significant variables for OHSS precaution (11). In the present study, the authors observed no difference in age, estradiol, testosterone, FSH and insulin in PCOS patients suffering from OHSS, when compared to PCOS women without OHSS. A reverse correlation between BMI and propensity for OHSS in PCOS patients was identified, most of OHSS patients had BMI $<24$ and it is possible that these patients are at greater risk to develop OHSS. This difference may somewhat explain the high incidence of OHSS, since it is more frequent in lean patients. Further analysis demonstrated the AUC value of BMI was 0.729, the sensitivity and specificity were 0.833 and 0.787 , respectively. However, obese women have been presented to be more prone to anovulation, and symptoms and PCOS are aggravated by obesity (20). Thus, a larger cohort study is required to assess BMI as a predictor for OHSS in PCOS patients.

The LH/FSH ratio is another important baseline parameter for predicting the risk of facing a high ovarian response to $\operatorname{COS}(1,2)$. The authors observed a significantly higher LH level and LH/FSH ratio in OHSS recruits compared to mean serum levels of control patients in the present study. FSH and LH serve different physiological roles within ovulation and are required for follicular growth and estrogen secretion in the ovary $(21,22)$. The sensitivity and specificity of LH/FSH ratio for prediction of OHSS were 0.709 and 0.821 , respectively. The AUC value of LH/FSH for OHSS was 0.741, which is very close to the value of $\mathrm{BMI}(\mathrm{AUC}=0.821)$ found in the current study. Despite limited data on the role of hormonal in predicting OHSS, these markers have being increasingly utilized in predicting ovarian response to stimulation. AMH levels have been previously evaluated $(23,24)$ and the results obtained presented much promise. A previous study demonstrated that AMH levels can identify women at high risk of developing OHSS (sensitivity $90.5 \%$, specificity $81.3 \%$ ), as a useful predictor of developing OHSS (12).

The present work especially focused on the bioinformatics analysis of identified proteins. $19.6 \%$ of these proteins were from extracellular region of the cell, and $19.1 \%$ were from organelles. These proteins were involved in many biological processes, such as metabolic process, energy production and conversion, as well as lipid transport and metabolism. As the most common endocrine and metabolic disease in 
reproductive age spectrum, PCOS frequently develop metabolic complications, such as glucose intolerance, insulin resistance, hyperinsulinemia and dyslipidemia $(25,26)$. These abnormalities put PCOS patients at high risk of cardiovascular disease, obesity and type 2 diabetes at a young age. Previous experimental evidence have demonstrated the intimate connection between endocrine disrupting chemicals and metabolic disturbances through impaired normal lipid and glucose homeostasis $(27,28)$. Therefore, the authors speculate that metabolic disorders may be a key risk factor of OHSS incidence in lean PCOS women.

Haptoglobin, fibrinogen and lipoprotein lipase were chosen as candidate protein markers for the prediction of OHSS in the study. It was observed that all these three proteins are precise markers at discriminating OHSS. Haptoglobin is an inflammatory factor, its functions include in antioxidant activity, hemoglobin binding, acute-phase response, immune system process, arterial restructuring and vascular disease (29). Inflammatory factors as key OHSS risk factors may lead to the increase of vascular permeability, angiogenesis, protein-rich fluid accumulates in peritoneum, pleura and pericardial space (30). Fibrinogen is an acute phase protein and the marker of coagulation potential, which involves in blood coagulation, cell-matrix adhesion, innate immune response, platelet activation and positive regulation of exocytosis (31). Moreover, it can be a hemostatic agent (32), which may explain the formation of thrombosis in severe OHSS. A previous study had already revealed that OHSS patients present an imbalance to homeostasis and this was characterized by high levels of fibrinogen and inflammatory factors, such as interleukin-6 and tumor necrosis factor- $\alpha$ (33). The biological processes of lipoprotein lipase include heparin binding, which associated with patients who have a low BMI (34). Above all, the three proteins that have been selected for the present study have never been reported as a predictive marker of OHSS in PCOS patients, and their potential roles in OHSS occurrence deserve further studies.

In summary, the present study successfully identified diagnostic biomarkers that may have a role in predicting severe OHSS among PCOS patients. The proteins of haptoglobin, fibrinogen and lipoprotein lipase presented higher sensitivity and specificity in OHSS. The proteomic results reported in the current study may help to gain deeper insights into the pathophysiology of OHSS in PCOS patients. Future studies should assess the quality of these three proteins as serum biomarkers of OHSS in PCOS patients in a larger population.

\section{Acknowledgements}

The present work was supported by grants from Science and Technology Development Foundation Item of Nanjing Medical University (grant no. 2013NJMU141) and the Nanjing Medical Science and Technique Development Foundation of Nanjing Department of Health (grant no. YKK14131).

\section{References}

1. Smith V, Osianlis T and Vollenhoven B: Prevention of ovarian hyperstimulation syndrome: A review. Obstet Gynecol Int 2015 $514159,2015$.
2. Nastri CO, Teixeira DM, Moroni RM, Leitão VM and Martins WP: Ovarian hyperstimulation syndrome: Patho physiology, staging, prediction and prevention. Ultrasound Obstet Gynecol 45: 377-393, 2015

3. Kumar P, Sait SF, Sharma A and Kumar M: Ovarian hyperstimulation syndrome. J Hum Reprod Sci 4: 70-75, 2011.

4. Sukul SP, Pekelharing JE, van Hooff MH, van der Weiden RM and van Asten JC: Thrombosis in ovarian hyperstimulation syndrome. Ned Tijdschr Geneeskd 158: A7108, 2014 (In Dutch).

5. Halupczok J, Kluba-Szyszka A, Bidzińska-Speichert B and Knychalski B: Ovarian hyperstimulation caused by gonadotroph pituitary adenoma-review. Adv Clin Exp Med 24: 695-703, 2015.

6. Nouri K, Tempfer CB, Lenart C, Windischbauer L, Walch K, Promberger R and Ott J: Predictive factors for recovery time in patients suffering from severe OHSS. Reprod Biol Endocrinol 12: 59, 2014.

7. Guo JL, Zhang DD, Zhao Y, Zhang D, Zhang XM, Zhou CQ and Yao SZ: Pharmacologic interventions in preventing ovarian hyperstimulation syndrome: A systematic review and network meta-analysis. Sci Rep 6: 19093, 2016.

8. Miller I, Chuderland D, Ron-El R, Shalgi R and Ben-Ami I: GnRH agonist triggering modulates PEDF to VEGF ratio inversely to hCG in granulosa cells. J Clin Endocrinol Metab 100: E1428-E1436, 2015.

9. Ingman WV and Robertson SA: Transforming growth factor-beta1 null mutation causes infertility in male mice associated with testosterone deficiency and sexual dysfunction. Endocrinology 148: 4032-4043, 2007.

10. Herr D, Bekes I and Wulff C: Local renin-angiotensin system in the reproductive system. Front Endocrinol (Lausanne) 4: 150, 2013.

11. Ashrafi M, Bahmanabadi A, Akhond MR and Arabipoor A: Predictive factors of early moderate/severe ovarian hyper stimulation syndrome in non-polycystic ovarian syndrome patients: Astatisticalmodel.ArchGynecolObstet 292:1145-1152, 2015.

12. Ocal P, Sahmay S, Cetin M, Irez T, Guralp O and Cepni I: Serum anti-Müllerian hormone and antral follicle count as predictive markers of OHSS in ART cycles. J Assist Reprod Genet 28: 1197-1203, 2011.

13. Markel G, Imazio M, Koren-Morag N, Galore-Haskel G, Schachter J, Besser M, Cumetti D, Maestroni S, Altman A, Shoenfeld Y, et al: CEACAM1 and MICA as novel serum biomarkers in patients with acute and recurrent pericarditis. Oncotarget 7: 17885-17895, 2016.

14. Xu D, Li Y, Li X, Wei LL, Pan Z, Jiang TT, Chen ZL, Wang C, Cao WM, Zhang X, et al: Serum protein S100A9, SOD3, and MMP9 as new diagnostic biomarkers for pulmonary tuberculosis by iTRAQ-coupled two-dimensional LC-MS/MS Proteomics 15: 58-67, 2015.

15. Palioura E and Diamanti-Kandarakis E: Polycystic ovary syndrome (PCOS) and endocrine disrupting chemicals (EDCs). Rev Endocr Metab Disord 16: 365-371, 2015.

16. Golan A and Weissman A: Symposium: Update on prediction and management of OHSS. A modern classification of OHSS. Reprod Biomed Online 19: 28-32, 2009.

17. Nhi DM, Huy NT, Ohyama K, Kimura D, Lan NT, Uchida L, Thuong NV, Nhon CT, Phuc le H, Mai NT, et al: A proteomic approach identifies candidate early biomarkers to predict severe dengue in children. PLoS Negl Trop Dis 10: e0004435, 2016.

18. Zhang Y, Kang Y, Zhou Q, Zhou J, Wang H, Jin H, Liu X, Ma D and Li X: Quantitative proteomic analysis of serum from pregnant women carrying a fetus with conotruncal heart defect using isobaric tags for relative and absolute quantitation (iTRAQ) labeling. PLoS One 9: e111645, 2014.

19. Chen L, Gu H, Li J, Yang ZY, Sun X, Zhang L, Shan L, Wu L, Wei X, Zhao Y, et al: Comprehensive maternal serum proteomics identifies the cytoskeletal proteins as non-invasive biomarkers in prenatal diagnosis of congenital heart defects. Sci Rep 6: 19248, 2016.

20. Ezeh U, Yildiz BO and Azziz R: Referral bias in defining the phenotype and prevalence of obesity in polycystic ovary syndrome. J Clin Endocrinol Metab 98: E1088-E1096, 2013.

21. Ezcurra D and Humaidan P: A review of luteinising hormone and human chorionic gonadotropin when used in assisted reproductive technology. Reprod Biol Endocrinol 12: 95, 2014 
22. Lehert P, Kolibianakis EM, Venetis CA, Schertz J, Saunders H, Arriagada P, Copt S and Tarlatzis B: Recombinant human follicle-stimulating hormone (r-hFSH) plus recombinant luteinizing hormone versus $\mathrm{r}$-hFSH alone for ovarian stimulation during assisted reproductive technology: Systematic review and meta-analysis. Reprod Biol Endocrinol 12: 17, 2014.

23. Peluso C, Fonseca FL, Gastaldo GG, Christofolini DM, Cordts EB, Barbosa $\mathrm{CP}$ and Bianco B: $\mathrm{AMH}$ and AMHR2 polymorphisms and AMH serum level can predict assisted reproduction outcomes: A cross-sectional study. Cell Physiol Biochem 35: 1401-1412, 2015.

24. Salmassi A, Mettler L, Hedderich J, Jonat W, Deenadayal A, von Otte S, Eckmann-Scholz C and Schmutzler AG: Cut-off levels of anti-mullerian hormone for the prediction of ovarian response, in vitro fertilization outcome and ovarian hyperstimulation syndrome. Int J Fertil Steril 9: 157-167, 2015.

25. Sam S: Adiposity and metabolic dysfunction in polycystic ovary syndrome. Horm Mol Biol Clin Investig 21: 107-116, 2015.

26. Carreau AM and Baillargeon JP: PCOS in adolescence and type 2 diabetes. Curr Diab Rep 15: 564, 2015.

27. Polyzos SA, Kountouras J, Deretzi G, Zavos C and Mantzoros CS The emerging role of endocrine disruptors in pathogenesis of insulin resistance: A concept implicating nonalcoholic fatty liver disease. Curr Mol Med 12: 68-82, 2012.
28. Alonso-Magdalena P, Quesada I and Nadal A: Endocrine disruptors in the etiology of type 2 diabetes mellitus. Nat Rev Endocrinol 7: 346-353, 2011.

29. Levy AP, Asleh R, Blum S, Levy NS, Miller-Lotan R, Kalet-Litman S, Anbinder Y, Lache O, Nakhoul FM, Asaf R, et al: Haptoglobin: Basic and clinical aspects. Antioxid Redox Signal 12: 293-304, 2010.

30. Orvieto R, Dratviman-Storobinsky O, Lantsberg D, Haas J, Mashiach R and Cohen Y: Interleukin-2 and SOCS-1 proteins involvement in the pathophysiology of severe ovarian hyperstimulation syndrome-a preliminary proof of concept. J Ovarian Res 7: 106, 2014.

31. Elliott BM and Aledort LM: Restoring hemostasis: Fibrinogen concentrate versus cryoprecipitate. Expert Rev Hematol 6: 277-286, 2013.

32. Wang S, Moustaid-Moussa N, Chen L, Mo H, Shastri A, Su R, Bapat P, Kwun I and Shen CL: Novel insights of dietary polyphenols and obesity. J Nutr Biochem 25: 1-18, 2014.

33. Chistyakova GN, Remizova II, Gazieva IA and Chermyaninova OV: Immunological and hemostasiological disorders in women with ovarian hyperstimulation syndrome. Gynecol Endocrinol 30 (Suppl 1): S39-S42, 2014.

34. Olivecrona G: Role of lipoprotein lipase in lipid metabolism. Curr Opin Lipidol 27: 233-241, 2016. 